\title{
Thermodynamic Analysis of the Irreversibilities in Solar Absorption Refrigerators
}

\author{
Emma Berrich Betouche ${ }^{1}$, Ali Fellah ${ }^{2}$, Ammar Ben Brahim ${ }^{2}$, Fethi Aloui ${ }^{3, *}$ and Michel Feidt ${ }^{4}$ \\ 1 GEPEA-UMR CNRS 6144 Laboratory, École des Mines de Nantes, Energetic Systems and Environment \\ Department, 4 Alfred Kastler Street BP 20722, 44307 Nantes, France; emna.berrich@univ-nantes.fr \\ 2 Applied Thermodynamics Research Unit (99/UR/11-21), National Engineering School of Gabès, Omar Ibn \\ Elkhattab Street, 6029 Gabès, Tunisia; al.fellah@gmail.com (A.F.); Ammar.Benbrahim@enig.rnu.tn (A.B.B.) \\ 3 LAMIH CNRS UMR 8201, Department of Mechanics, Valenciennes University, Campus Mont Houy, \\ F-59313 Valenciennes Cedex 9, France \\ 4 LEMTA, CNRS UMR 7563, ENSEM-INPL, Université de Lorraine, 2 Avenue de la Forêt of Haye BP 160, \\ 54504 Vandoeuvre-lès-Nancy, France; michel.feidt@univ-lorraine.fr \\ * Correspondence: Fethi.aloui@univ-valenciennes.fr; Tel.: +33-3-27-51-19-62; Fax: +33-3-27-51-19-61
}

Academic Editor: Kevin H. Knuth

Received: 29 October 2015; Accepted: 22 March 2016; Published: 24 March 2016

\begin{abstract}
A thermodynamic analysis of the irreversibility on solar absorption refrigerators is presented. Under the hierarchical decomposition and the hypothesis of an endoreversible model, many functional and practical domains are defined. The effect of external heat source temperature on the entropy rate and on the inverse specific cooling load (ISCL) multiplied by the total area of the refrigerator $A / Q_{e}$ are studied. This may help a constructor to well dimension the solar machine under an optimal technico-economical criterion $A / Q_{e}$ and with reasonable irreversibility on the refrigerator. The solar concentrator temperature effect on the total exchanged area, on the technico-economical ratio $A / Q_{e}$, and on the internal entropy rate are illustrated and discussed. The originality of these results is that they allow a conceptual study of a solar absorption refrigeration cycle.
\end{abstract}

Keywords: irreversibility; entropy; inverse specific cooling load; technico-economical criterion

\section{Introduction}

Knowledge of the entropy transfer is of capital interest to better understanding refrigerator behavior and to ensuring the control of the design processes and performances. Since irreversibility controls the entropy generation and thus determines the performance of solar absorption refrigerators, the entropy generation constitutes the purpose of analysis adopted by several studies. Theoretical and experimental studies concerning heat transfer irreversibility remain topical, and even the best elaborated models are still unable in certain cases to restore experimental reality to their predictions.

Several approaches, based on numerical and experimental studies, have been proposed to undertake the irreversibility for the determination of real cycle's behaviour [1-5]. The usefulness of thermodynamics to develop more significant upper bounds for various optimization criteria concerning reverse cycle machines and their possible interrelation was reviewed in [6]. The importance of irreversibility in the characterization of reverse cycle machines was also highlighted. The studies carried out in [7] on the heat transfer effect relative to the irreversibility on the specific cooling load (SCL) of refrigerators permit the distinction between four types of thermodynamic models relative to four forms of irreversibility (reversible, endoreversible, exoreversible, and irreversible). Bejan [8] studied the internal heat transfer irreversibility for refrigeration plants. He introduced the heat leak conductance between ambiance and the refrigerated volume (essentially thermal insulation) to calculate the heat flux as a conductance multiplied by the heat and cold source temperature 
difference. As a result, with three-heat transfer irreversibility, an equal-partition of both heat and cold compartment conductance was obtained. A complementary study was proposed by a thermo-economics approach. In 1993, one researcher completed a calculation for minimizing heat exchanger inventory in power or refrigeration plants [9]. In 1996, another researcher proposed a method of entropy generation minimization, or modelling and optimization, based on combined heat transfer and thermodynamics [10]. In fact, the thermodynamic minimization of the entropy production constitutes a good method of modeling real processes, i.e., irreversible machines. The models integrate the thermodynamic-based principles, the heat transfer, and the optimization, which were adjusted to constraints related to finite dimensions and finite times.

When the absorption cycle operates under steady-state conditions, the net entropy transfer from the working fluid to the heat reservoirs is equal to the internal entropy generation in the working fluid [11].

The inverse specific cooling load (ISCL), $A / Q_{e}$, is frequently used in industrial engineering optimization. It represents the physical phenomena that take place in refrigeration systems. It is considered a universal criterion for these systems [12]. Fellah et al. [4] proposed a relationship between this criterion - the refrigerator area multiplied by the inverse neat cooling load (INCL) and the inverse of the coefficient of performance (COP). They proposed an optimal operational zone of refrigerators that can be chosen in specific intervals. Indeed, they obtained an interesting curve of the performance function of the technico-economical criterion $\left(A / Q_{e}\right)$ for different values of the external heat source temperatures and thermal conductance of the solar absorber. The different curves are formed by three branches. The first branch is located in the region of low values of the COP. Here, the COP decreases when the exchange area increases owing to the increase of heat losses in the heat transfer process. This could be used as a malfunctioning criterion. This is the case where the condenser and the generator temperatures increase, getting close to the heat source temperature. This branch of the curves is of academic interest [13]. The part of these curves of practical interest is the upper region, which corresponds to high values of COP. In fact, the vertical branch obtained by Fellah et al. [4] shows a speedy variation of COP for a little variation of the ISCL. The lower heat-exchange area with the higher thermal load in the evaporator can be deduced from the left limit of this branch. Moreover, the most significant result that can be deduced is the determination of the constraint related to the minimal total area of the absorption refrigeration unit. They found that the COP variation function of the endoreversible technico-economical criterion $A / Q_{e}$ is lower than the one derived by Berlitz et al. [14], which is between 0.3 and $0.6 \mathrm{~m}^{2} / \mathrm{kW}$. They proposed an optimal operating work zone limited by:

$$
\begin{gathered}
0.3<\text { COP }<1 \\
0.02 \mathrm{~m}^{2} / \mathrm{kW}<A / Q_{e}<0.1 \mathrm{~m}^{2} / \mathrm{kW}
\end{gathered}
$$

As previous works proposed the study of the effect on $A / Q_{e}$ on the performances, and that these performances are function of the irreversibilities, we propose in this paper the same results for the effect of $A / Q_{e}$ on the entropy.

\section{Thermodynamic Approach and Hierarchical Decomposition}

The solar absorption refrigeration cycle is presented in Figure 1. It is composed of a solar concentrator, i.e., the solar collector (heat source), a thermal solar converter (TE1), an intermediate source, a cold source, and four main elements: a generator, an absorber, a condenser, and an evaporator. The generator and the absorber constitute a thermal engine TE2, while the condenser and the evaporator form the thermal receptor TR. The exchanged fluxes and powers that reign in the different compartments of the machine are mentioned in Figure 1. The parameterization of the installation comprises fluxes and powers as well as temperatures reigning in the different compartments of the machine. The $P_{r e f}$ is the refrigeration power. 


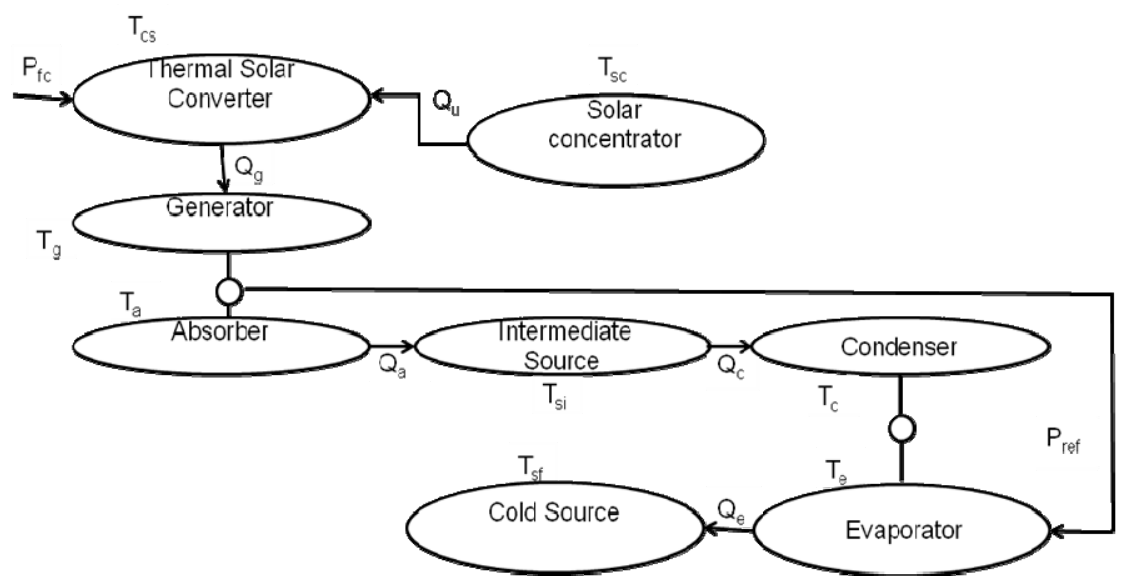

Figure 1. Composition of a solar absorption refrigerator.

The hierarchical decomposition, the optimization by sub-structuring [15], and the approaches which combine thermodynamic criteria and technico-economical criteria [16] for the study of the solar absorption cycle, which are well developed in $[4,5,16]$, were applied to the absorption refrigeration cycle. The procedure and base conditions used for the solar system and absorption cycle have been described in our previous papers $[17,18]$. The thermodynamic analysis of the irreversibility on refrigerators, using the hierarchical decomposition, is presented in this paper.

The hierarchical decomposition of the solar absorption refrigeration cycle is illustrated in Figure 2. The four subdivision levels of the hierarchical decomposition are: the first level (L.I), which is the compact global system (TE1 + TE2 + TR). It is divided into two sublevels (L.II.1 and 2): the thermal converter (TE1), and the command and refrigeration system.

The command and refrigeration system is also decomposed in two sublevels: the thermal engine TE2 and the thermal receptor TR. The thermal engine TE2 is composed of the generator and the absorber. The thermal receptor TR is constituted by the condenser and the evaporator.

The four principle elements of an absorption refrigeration cycle (generator, absorber, condenser, and evaporator) constitute the last level (L.IV).

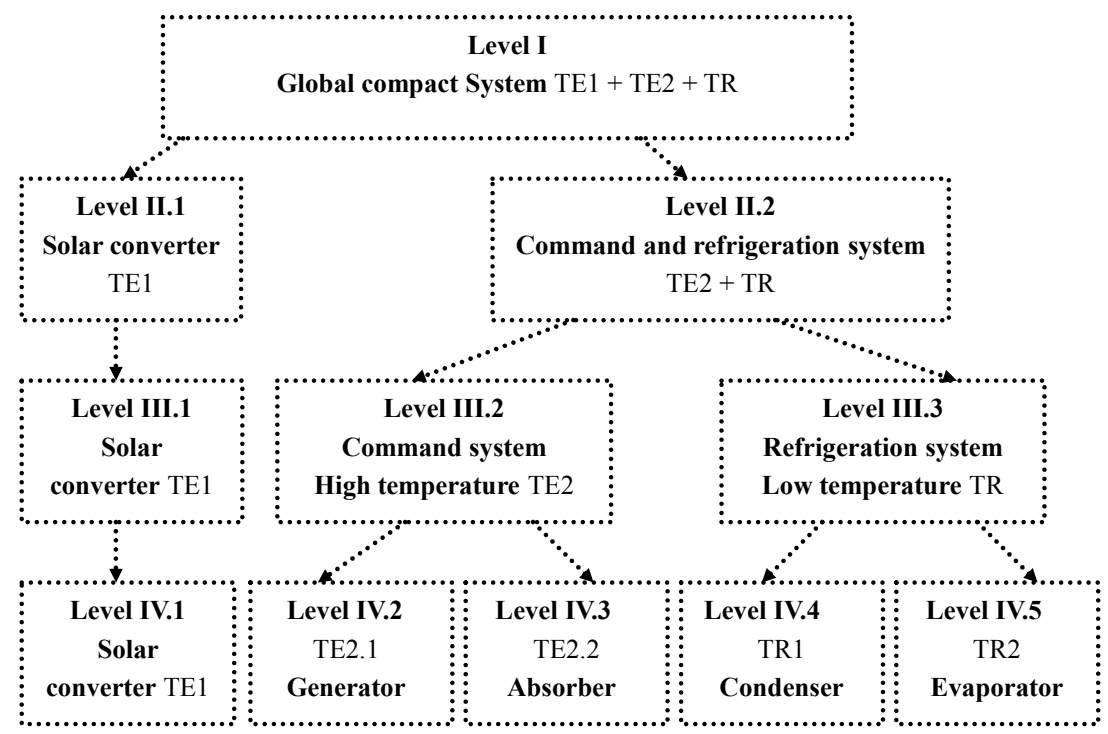

Figure 2. Hierarchical decomposition of the solar absorption refrigeration cycle. 
Considering the reversibility inside the system, the first thermodynamic law $[4,19,20]$ could be written for the command and refrigeration cycle as:

$$
Q_{e}+Q_{g}-Q_{a}-Q_{c}=0
$$

The second thermodynamic law can be written as:

$$
\frac{Q_{e}}{T_{e}}+\frac{Q_{g}}{T_{g}}-\frac{Q_{a}}{T_{a}}-\frac{Q_{c}}{T_{c}}+S=0
$$

where $S$ is the entropy production rate.

The Lagrange multiplier method requires an objective function and constraints.

According to the functional and conceptual unknowns' apparition, the study concerns a sublevel, a level, or a set of sublevels [17].

For an absorption refrigeration cycle comprised of a generator, an absorber, an evaporator, and a condenser, the coefficient of performance can be estimated as per $[5,10]$ :

$$
C O P=\frac{Q_{e}}{Q_{g}}=\frac{P_{r e f}}{Q_{g}} \frac{Q_{e}}{P_{r e f}}=\eta_{T E 2} \eta_{T R}
$$

where $\eta_{T E 2}$ : the command system High temperature TE2 efficiency (generator and absorber); and $\eta_{T R}$ : the refrigeration system Low temperature TR efficiency (condenser and evaporator).

The heat exchange areas of the command system High temperature TE2, the refrigeration system Low temperature TR, and the total exchange area are, respectively:

$$
\begin{gathered}
A_{h}=A_{g}+A_{a} \\
A_{r}=A_{c}+A_{e} \\
A=A_{h}+A_{r}
\end{gathered}
$$

The command system High temperature TE2 efficiency can be written as per [5]:

$$
\eta_{T E 2}=1-\frac{T_{s i}}{T_{s c}-\frac{Q_{g}}{A_{h} U_{h}}}
$$

where:

$$
U_{h}=\frac{U_{g} U_{a}}{\left(\sqrt{U_{g}}+\sqrt{U_{a}}\right)^{2}}
$$

Additionally, the refrigeration system Low temperature TR efficiency can be written as per [5]:

$$
\eta_{T R}=\frac{T_{s f}-\frac{Q_{e}}{U_{r} A_{r}}}{T_{s i}-T_{s f}+\frac{Q_{e}}{U_{r} A_{r}}}
$$

where:

$$
U_{r}=\frac{U_{e} U_{c}}{\left(\sqrt{U_{e}}+\sqrt{U_{c}}\right)^{2}}
$$


The coefficient of performance can be written as:

$$
C O P=\left\{1-\frac{T_{s i}}{T_{s c}-\frac{Q_{g}}{A_{h} U_{h}}}\right\} \frac{T_{s f}-\frac{Q_{e}}{U_{r} A_{r}}}{T_{s i}-T_{s f}+\frac{Q_{e}}{U_{r} A_{r}}}
$$

The entropy production can be written as:

$$
S=\frac{Q_{a}+Q_{c}}{T_{s i}}-\frac{Q_{e}}{T_{e}}-\frac{Q_{g}}{T_{g}}
$$

The optimization Lagrange function can be written, for every level and sub-level, as:

$$
L=Q_{j}+\lambda_{i}\left(\sum Q_{i}\right)+\lambda_{j}\left(\sum \frac{Q_{i}}{T_{i}}\right)
$$

where $\lambda$ is the Lagrange coefficient.

We applied the hierarchical decomposition and the optimization by sub-structuring [15] and approaches that combine thermodynamic criteria and technical-economic criteria [16] to study the solar absorption cycle. Interesting relations were obtained. It allows the study of external temperatures on the entropy production, performances, and area effect $[17,18]$. We found for example that the work fluid temperature on the condenser is equal to the absorber one [17]. This is in conformity with [3-5]:

$$
T_{c}=T_{a}=T_{s i}
$$

In this paper, we investigated the effect of external temperatures on the entropy production. The results are presented for a $T_{s c}$ range of $80-120^{\circ} \mathrm{C}$ for a fixed cold source and intermediate source temperatures: $T_{s f}=10{ }^{\circ} \mathrm{C}$ and $T_{s i}=28^{\circ} \mathrm{C}$. As it is directly related to the cycle performances, we studied the entropy production function of the technical-economic criteria $A / Q_{e}$ in order to compare it to the $A / Q_{e}$ optimal range found in [14].

\section{Results and Discussion}

\subsection{Solar Concentrator Temperature Effect on the Area by Inverse Specific Cooling Load (ISCL) and on Irreversibility}

One of the most important aims of energetic researchers and refrigerator constructors is generally to find a compromise between the $\left(A / Q_{e}\right)$ which is a technico-economical criterion for refrigerators and heat pumps, i.e., the total exchanged area of the refrigerator divided by the specific cooling load $Q_{e}$, and the entropy takes minimal losses, due to system irreversibility, with few dimensions of the installation, thus allowing an increase in the performances of the machine. The effect of the solar concentrator temperature $T_{s c}$ on the inverse specific cooling load (ISCL) multiplied by the total area of the refrigerator, i.e., $A / Q_{e}$, and on the internal entropy rate is illustrated in Figure 3. As we can see, from the curves $\left(T_{s c}=80^{\circ} \mathrm{C}, 85^{\circ} \mathrm{C}, 90^{\circ} \mathrm{C}, 95^{\circ} \mathrm{C}, 100^{\circ} \mathrm{C}, 105^{\circ} \mathrm{C}, 110^{\circ} \mathrm{C}, 150^{\circ} \mathrm{C}\right.$, and $\left.120^{\circ} \mathrm{C}\right)$, for a fixed cold source and intermediate source temperatures $\left(T_{s f}=10^{\circ} \mathrm{C}\right.$ and $\left.T_{s i}=28^{\circ} \mathrm{C}\right)$, the total exchanged area $A$ and the internal entropy rate can be easy deduced for a specific cooling load $Q_{e}$ and a work temperature $T_{s c}$. For example, to construct a solar absorption refrigeration machine assuring a total exchanged area of $28.5 \%$ of the specific cooling load $Q_{e}$, for a heat source temperature $T_{s c}$ bigger than $105^{\circ} \mathrm{C}$, we estimate irreversibility values less than $0.265 \mathrm{~kW} / \mathrm{K}$.

For every case, functional and practical domains are defined. Indeed, we proved that, in the range of heat source temperature considered from $80{ }^{\circ} \mathrm{C}$ to $120^{\circ} \mathrm{C}$, which is the practical zone of work, a constructor can define the optimal technico-economical ratio of a refrigerator $A / Q_{e}$ and its irreversibility for a heat source temperature work range. 


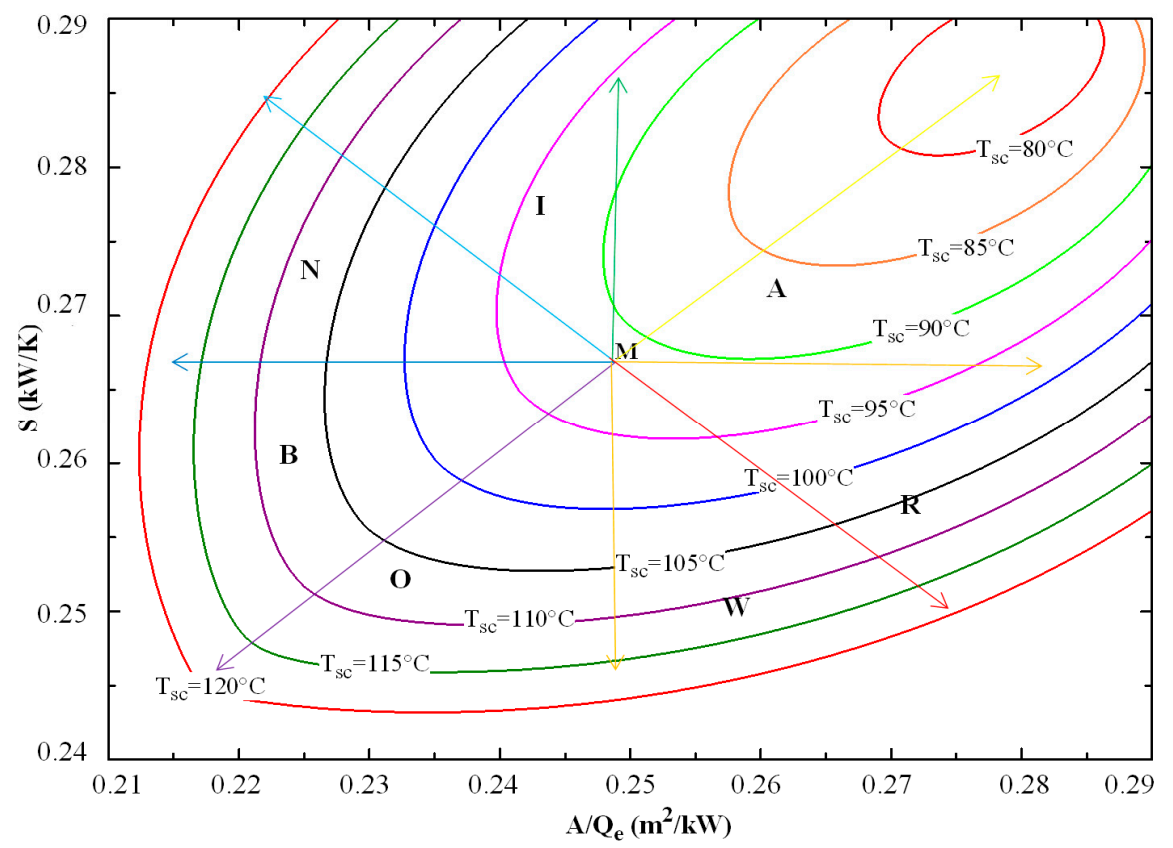

Figure 3. Effect of the solar concentrator temperature on the technico-economical ratio $A / Q_{e}$ and on the internal entropy rate for $T_{s f}=10^{\circ} \mathrm{C}$ and $T_{s i}=28^{\circ} \mathrm{C}$.

The optimization gives mathematically two solutions. The optimal operational zone of the refrigerators is to be chosen in the intervals corresponding to minimized internal entropy rate which corresponds to optimal COP. Consider a work state described by point $\mathrm{M}$ in our optimal domain. This state is initially characterized by a heat source temperature equal to approximately $92{ }^{\circ} \mathrm{C}$, a technico-economical criterion corresponding to a total surface equals to $24.9 \%$ of the specific cooling load (SCL) value, and an internal entropy rate of $0.267 \mathrm{~kW} / \mathrm{K}$. We composed our optimal domain to seven zones ( $R, A, I, N, B, O, W)$, which have as an origin our initial state " $M$ ", and we tried to determine the suitable and optimal work zone. From a clockwise direction, every zone is characterized by two successive colors of the "rainbow". This "rainbow" schema allow us to distinguish non-suitable work domains from suitable ones by analogy to our domain of visibility, and our suitable and optimal work domain will be characterized by the region between the line color 1 and line color 2 . In fact:

- Zone " $\mathrm{R}$ ": This is limited by the red line and the orange line (pointer directions having the initial state $\mathrm{M}$ as an origin) and characterized by a decrease in the internal entropy rate as the heat source temperature of work increases. These results are logical and expected because, when the heat source temperature of work increases, the COP increases, and as we know the performances of the machine become more interesting when the irreversibility decreases. These results are in conformity with Fellah et al.'s [4] analysis. This region of work is not a suitable one for a constructor or an experimenter, as they will be obliged to increase the total area about $9.1 \%$ of the specific cooling load (SCL) and the heat source temperature of work more than $25^{\circ} \mathrm{C}$ in order to decrease the internal entropy rate approximately $7.5 \%$.

- Zone " $\mathrm{A}$ ": This is limited by the orange line and the yellow line and characterized by an increase in the internal entropy rate as heat source work temperature from the initial state decreases, i.e., $92{ }^{\circ} \mathrm{C}$ to less than $80^{\circ} \mathrm{C}$. In fact, when the heat source work temperature decreases, the COP decreases and thus the irreversibility increases.

- Zone "I": This is between the green line and the light blue line and characterized by an increase in the internal entropy rate as the heat source temperature increases. The point of merit here for a constructor is the reduction of the total area, which decreases more than $2.5 \%$ of the initial state of 
work, but the internal entropy rate increases when $T_{s c}$ increases. For the experimenter, other work regions may be more interesting.

- Zone " $\mathrm{N}$ ": This zone presents a critical case. It is between the light blue line and the dark blue line. It is characterized by a vertical temperature curves for low $T_{s c}$ and slightly inclined ones for high $T_{s c}$. Indeed, it is characterized by a maintained fixed economic criterion for low source temperature and an internal entropy rate variation range limited to a maximum of $2 \%$ and a slight increase in the technico-economical criterion values for high values of the heat source temperature with an internal entropy rate variation that can attend $6.9 \%$.

- Zone " $\mathrm{B}$ ": This is between the dark blue line and the indigo line. It is characterized by slightly inclined temperature curves for low $T_{s c}$ and vertical ones for high $T_{s c}$, contrary to the previous zone. Indeed, the technico-economical criterion is maintained fixed for a high source temperature; the internal entropy rate variation range can attend a maximum of $8.24 \%$. For low values of the heat source temperature, the technico-economical criterion slightly increases to approximately the same extent for all work temperatures (1.27\%). This small increase is accompanied by an internal entropy rate variation that can attend $1.7 \%$. As we can see, in this zone of work, the internal entropy rate can be decreased by increasing heat source temperature. Thus, it may be a good region of work.

- Zone "O": This represents a suitable work zone. It is between the indigo line and the purple line and characterized by a decrease in the internal entropy rate while heat source temperature increases. In fact, when the heat source temperature increases, the irreversibility decreases and the COP increases. The advantage of working in this zone is that we simultaneously reduce the refrigerator area and the irreversibility and then increase the performances of our solar machine. We minimize the irreversibility more than $8 \%$ and the total area more than $13 \%$.

- Zone " $\mathrm{W}$ ": This is between the purple line and the red line and characterized by a decrease in the internal entropy rate while heat source temperature increases. These results are logical and predictable because when the heat source temperature of work increases, the irreversibility decreases, i.e., the performances of the solar absorption refrigerator become more important. For high values of the heat source temperature, when we increase the temperature from our initial state to $120^{\circ} \mathrm{C}$ for example, the internal entropy rate decreases about $8.16 \%$. This work zone is characterized by a slightly inclined temperature curves for low $T_{s c}$. For a fixed source temperature $\left(T_{s c}=100{ }^{\circ} \mathrm{C}\right.$ for example), the internal entropy slightly increases while the technico-economical criterion increases.

Please note that the zone between A and I, which is between the yellow line and the green line, is characterized by an increase in the internal entropy rate while heat source temperature decreases. Indeed, the COP decreases when the heat source temperature decreases.

As we can see, for every work zone, there is a critical case characterized by a fixed internal entropy rate or a fixed technico-economical criterion as if it seems to be analogical to a transition phase, between a domain and another one. Indeed, according to the straight line drawn horizontally to all the curves of the different cases, it is possible, for example, to decrease the total surface to approximately $16 \%$ of the specific cooling load (SCL) while increasing the heat source temperature to approximately $25^{\circ} \mathrm{C}$, while the internal entropy rate remains constant.

It should be noted that, even if we encourage work in one zone more than another, all the "rainbow" domains are generally good as they are in a good range, i.e., $0.21<A / Q_{e}<0.29 \mathrm{~m}^{2} / \mathrm{kW}$, slightly lower than that derived by Berlitz et al. [14] for the technico-economical criterion, which is $0.3<A / Q_{e}<0.6 \mathrm{~m}^{2} / \mathrm{kW}$. According to Fellah et al.'s [4] work, a variation of $70 \%$ in the performance is related to a variation of $80 \%$ on the technico-economical criterion. In our case of study, a variation of $27.6 \%$ on the technico-economical criterion is related to a variation in the internal entropy rate of approximately $17 \%$ for $T_{s c}$ working range, and $T_{s f}=10^{\circ} \mathrm{C}$ and $T_{s i}=28^{\circ} \mathrm{C}$. 
A good constructor or/and experimenter usually seeks an economic finality, which is generally related to minimizing the under construction machine's area, or an optimal finality, which is related to minimizing machine irreversibility. Some general remarks can be helpful:

A decrease in the total area of $22.4 \%$ is possible, and an increase in $12 \%$ of the irreversibility may be attainable, for an identical heat source temperature of work $T_{s c}$; for example, for $T_{s c}=105^{\circ} \mathrm{C}$.

To fulfill fewer irreversibilities on the system (for example, a decrease in $15 \%$ of the irreversibility), which is accompanied with a decrease in the area of $24.5 \%$, an increase in the work temperature $T_{s c}$ of approximately $40^{\circ} \mathrm{C}$ is recommended (from $T_{s c}$ equals $80^{\circ} \mathrm{C}$ to $120^{\circ} \mathrm{C}$, for example).

A point of merit can be announced. An interesting new endoreversible technical and economic criterion regarding the optimal operating region of the refrigeration systems can be formulated as follows:

$$
0.24 \mathrm{~m}^{2} / \mathrm{kW}<A / Q_{e}<0.267 \mathrm{~m}^{2} / \mathrm{kW}
$$

These deductions can be useful for optimal conception of solar absorption refrigerators.

\subsection{Solar Concentrator Temperature Effect on the Total Exchanged Area and Entropy}

The hot heat source temperature $T_{s c}$ effect on the total exchanged area and on the internal entropy rate was studied. A plot of the variation of the total area, given by Equation (8), with the heat source temperature, for different values of the internal entropy rate, for $T_{s f}=10^{\circ} \mathrm{C}$ and $T_{s i}=28^{\circ} \mathrm{C}$, is shown in Figure 4. The different curves allow for the deducing, for a work temperature $T_{s c}$, of the total exchanged area and the endoreversible irreversibility created under the machine.

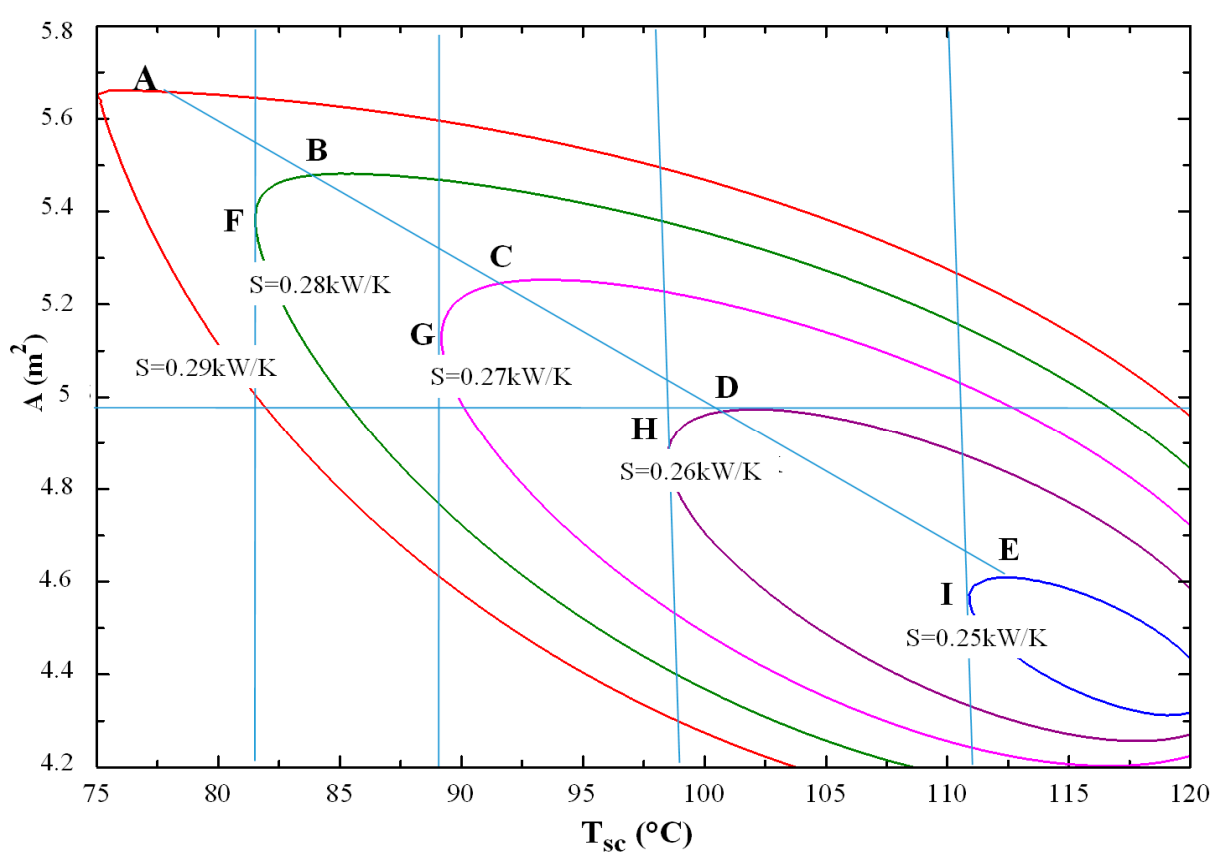

Figure 4. Effect of the solar concentrator temperature $T_{S c}$ on the total exchanged area and the internal entropy rate for $T_{s f}=10^{\circ} \mathrm{C}$ and $T_{s i}=28^{\circ} \mathrm{C}$.

The intersection between all the entropies curves and their horizontal tangents allows for the determination of the maximums of refrigerator area allowed for each heat source temperature and the appropriated endoreversible irreversibility expected. All of these maximums are aligned. The line regrouped these maximums of area divides the domain into two regions. Indeed, it corresponds to the principle domain separation line, which allows us to distinguish between two principle domains: The first one is characterized by a proportional variation of the entropies with heat source temperature for fixed total refrigerator area, while the second domain is characterized by an inversely proportional 
internal entropy rate variation for fixed refrigerator area. Consider point $\mathrm{D}$, for example, of this principle domain separation line. It can be seen that this point corresponds to the maximum total area for a work temperature $T_{s c}$, equal to approximately $101^{\circ} \mathrm{C}$. The internal entropy rate is equal to $0.26 \mathrm{~kW} / \mathrm{K}$. It is the intersection between the corresponded internal entropy rate curve and its horizontal tangent. The tangent passing from $\mathrm{D}$ (i.e., for a fixed refrigerator area) divides the domain to two regions: When we increase the heat source temperature, the internal entropy rate increases (first domain); and, from the other side of point $\mathrm{D}$, for a fixed area, when we decrease $T_{s c}$, the internal entropy rate decreases (second domain). We remarked that the horizontal distances between two successive internal entropy rate curves correspond to an identical temperature variation of approximately $11^{\circ} \mathrm{C}$ and an internal entropy rate variation of $1 \%$ : This corresponds to the internally reversible cycle.

We deduced that, on the optimal work region, for high heat source temperatures $T_{s c}$ values, the internal entropy rate becomes smaller with acceptable areas. For example, to construct a solar frigorific machine assuring a total exchanged area of $4.4 \mathrm{~m}^{2}$, a decrease in about $14 \%$ of the internal entropy rate is predicted for an increase in approximately $20^{\circ} \mathrm{C}$ on the heat source temperature.

\section{Conclusions}

The internal entropy rate production is strongly related to the cycle performances. A solar refrigerator conceptual design is proposed here, taking into consideration the irreversibility impact on the machines performances. Optimal conceptual and functional parameters were coupled into interesting relations. The effect of the external temperature and the specific cooling load or the technico-economical criterion $A / Q_{e}$ on the internal entropy rate and the machine area were studied, allowing a definition of optimal work regions. An interesting new endoreversible technical and economic criterion was proposed: $0.24 \mathrm{~m}^{2} / \mathrm{kW}<A / Q_{e}<0.267 \mathrm{~m}^{2} / \mathrm{kW}$. A variation of $27.6 \%$ on the technico-economical criterion is related to a variation of the internal entropy rate of approximately $17 \%$ for $T_{s c}$ working range, and $T_{s f}=10^{\circ} \mathrm{C}$ and $T_{s i}=28^{\circ} \mathrm{C}$. The features of this work are important. They concern an optimal establishment with a simple but universal method. It passes in the research's disciplinary decentralization.

Acknowledgments: This work was supported by the National Engineering School of Gabés in Tunisia.

Author Contributions: Emma Berrich Betouche realized this work, analyzed the results and wrote the paper. Ammar Ben Brahim, Ali Fellah, Michel Feidt and Fethi Aloui contributed to the results analysis and the paper revision. All authors have read and approved the final manuscript.

Conflicts of Interest: The authors declare no conflict of interest.

\section{References}

1. Feidt, M. Thermodynamics applied to reverse cycle machines, a review. Int. J. Refrig. 2010, 33, 1327-1342. [CrossRef]

2. Chen, J. The equivalent cycle system of an endoreversible absorption refrigerator and its general performance characteristics. Energy 1995, 20, 995-1003. [CrossRef]

3. Wijeysandera, N.E. Performance of three-heat-reservoir absorption cycles absorption cycles with external and internal irreverbilities. Appl. Therm. Eng. 1997, 17, 1151-1161. [CrossRef]

4. Fellah, A.; Ben Brahim, A.; Bourouis, M.; Coronas, A. Cooling loads analysis of an equivalent endoreversible model for a solar absorption refrigerator. Int. J. Exergy 2006, 3, 452-465. [CrossRef]

5. Fellah, A. Intégration de la Décomposition Hiérarchisée et de l'Endoréversibilité dans l'étude d'un Cycle de Réfrigeration par Absorption Solaire: Modélisation et Optimisation. Ph.D. Thesis, National Engineering School of Tunis, University of Tunis-El Manar, Tunis, Tunisia, 2008. (In French).

6. Petre, C.; Feidt, M.; Costea, M.; Petrescu, S. A model for study and optimization of real operating refrigeration machines. Int. J. Energy Res. 2009, 33, 173-179. [CrossRef]

7. Crosu, L.; Feidt, M.; Benelmir, R. Study of the improvement in the performance coefficient of machines operating with three reservoirs. Int. J. Exergy 2004, 1, 147-162. 
8. Bejan, A. Theory of heat transfer irreversible refrigeration plants. Int. J. Heat Mass Transf. 1989, 32, 1631-1639. [CrossRef]

9. Bejan, A. Power and refrigeration plants for minimum heat exchanger inventory. J. Energy Resour. Technol. 1993, 115, 148-150. [CrossRef]

10. Bejan, A. Method of entropy generation minimization, or modeling and optimization based on combined heat transfer and thermodynamics. Revue Générale de Thermique 1996, 35, 637-646. [CrossRef]

11. Wijeysundera, N.E. An irreversible thermodynamic model for solar powered absorption cooling systems. Sol. Energy 2000, 68, 69-75. [CrossRef]

12. Gordo, J.M.; Ng, K.C. Predictive and diagnostic aspects of universal thermodynamic model for chillers. Int. J. Heat Mass Transf. 1995, 38, 807-818. [CrossRef]

13. Summerer, F. Evaluation of absorption cycles with respect to COP and economics. Int. J. Refrig. 1996, 19, 19-24. [CrossRef]

14. Berlitz, T.; Satzger, P.; Summerer, F.; Ziegler, F.; Alefeld, G. A contribution to the evaluation of the economic perspectives of absorption chillers. Int. J. Refrig. 1999, 220, 67-76. [CrossRef]

15. Meeuse, F.M. On the Design of Chemical Processes with Improved Controllability Characteristics; DUP Science: Delft, The Netherlands, 2002.

16. Berrich, E.; Fellah, A.; Ben Brahim, A.; Feidt, M. Etude Conceptuelle et Fonctionnelle d'un Cycle de Réfrigération Par Absorption Solaire Selon l'Approche d'Endoréversibilité. In Proceedings of the 17th Annual SFT Conference on Efficacité Energétique, Vannes, France; 2009; pp. 695-700. (In French).

17. Berrich, E.; Fellah, A.; Ben Brahim, A.; Feidt, M. Conceptual and functional study of a solar absorption refrigeration cycle. Exergy 2011, 8, 265-280. [CrossRef]

18. Berrich, E.; Fellah, A.; Ben Brahim, A.; Aloui, F.; Feidt, M. Hierarchical decomposition thermodynamic approach for the study of solar absorption refrigerators performances. Entropy 2016, 18, 82. [CrossRef]

19. Zheng, T.; Chen, L.; Sun, F.; Wu, C. Performance optimization of an irreversible four-heat-reservoir absorption refrigerator. Appl. Energy 2003, 76, 391-414. [CrossRef]

20. Feidt, M. Thermodynamique et Optimisation Énergétique des Systèmes et Procédés; Lavoisier: Paris, France, 1987. (In French)

(C) 2016 by the authors; licensee MDPI, Basel, Switzerland. This article is an open access article distributed under the terms and conditions of the Creative Commons by Attribution (CC-BY) license (http://creativecommons.org/licenses/by/4.0/). 